\begin{tabular}{|c|c|}
\hline Title & $\begin{array}{l}\text { Perioperative changes of the slope in the preload recruitable stroke work relationship by a single beat technique after } \\
\text { mitral valve surgery in functional mitral regurgitation with non-ischemic dilated cardiomy opathy }\end{array}$ \\
\hline Author(s) & $\begin{array}{l}\text { Ishigaki, Takahiro; Shingu, Y asushige; Katoh, Nobuy asu; W akasa, Satoru; Katoh, Hiroki; Ooka, Tomonori; Kubota, } \\
\text { Suguru; Matsui, Y oshiro }\end{array}$ \\
\hline Citation & $\begin{array}{l}\text { General Thoracic and Cardiovascular Surgery, 68, 30-37 } \\
\text { https://doi.org/10.1007/s11748-019-01164-w }\end{array}$ \\
\hline Issue Date & $2020-01$ \\
\hline Doc URL & http:/hdl.handle.net/2115/80089 \\
\hline Rights & $\begin{array}{l}\text { This is a post-peer-review, pre copyedit version of an article published in General thoracic and cardiovascular surgery } \\
\text { The final authenticated version is avail able online at: } h \text { ttp://dx.doi.org } / 10.1007 / / 11748-019-01164 \mathrm{w}\end{array}$ \\
\hline Type & article (author version) \\
\hline File Information & Gen Thorac Cardiovasc Surg_2019.pdf \\
\hline
\end{tabular}

Instructions for use 
Original article

\section{Perioperative Changes of the Slope in the Preload Recruitable Stroke Work Relationship by a Single-Beat Technique After Mitral Valve Surgery in Functional Mitral Regurgitation With Non-ischemic Dilated Cardiomyopathy}

Takahiro Ishigaki ${ }^{1}$, M.D., Yasushige Shingu ${ }^{1}$, M.D., Ph.D., Nobuyasu Katoh ${ }^{1}$, MD., PhD, Satoru Wakasa ${ }^{1}$, M.D., Ph.D., Hiroki Katoh², MD., Ph.D., Tomonori Ooka ${ }^{1}$, M.D., Ph.D., Suguru Kubota ${ }^{1}$, M.D., Ph.D., Yoshiro Matsui ${ }^{1}$, M.D., Ph.D.

1. Department of Cardiovascular and Thoracic Surgery, Hokkaido University Hospital, Sapporo, Japan

2. Emergency and Clinical Care Center, Hokkaido University Hospital, Sapporo, Japan

\section{Corresponding author:}

Yasushige Shingu, M.D., Ph.D.

Department of Cardiovascular and Thoracic Surgery,

Faculty of Medicine and Graduate School of Medicine, Hokkaido University

Kita-15, Nishi-7, Kita-ku, Sapporo 060-8638, Japan

Tel: +81-11-706-6042

Fax: +81-11-706-7612

E- mail: shingu@huhp.hokudai.ac.jp

Key words: Mitral regurgitation, dilated cardiomyopathy, preload recruitable stroke work relationship

*This study was presented at the Featured Abstracts Session in the 71st Annual Scientific Meeting of the Japanese Association for Thoracic Surgery on Oct 3-6, 2018 in Tokyo. 


\begin{abstract}
Objectives: The slope in the preload recruitable stroke work relationship is a highly linear, load-insensitive contractile parameter. However, the perioperative change of the slope has not been reported before. We examined the perioperative slope from a steadystate single beat in patients with functional mitral regurgitation and assessed the correlation with brain natriuretic peptide (BNP) levels.
\end{abstract}

Methods: The study included 16 patients with non-ischemic dilated cardiomyopathy and refractory heart failure: 10 patients underwent mitral valve plasty and left ventricular plasty (MVP+LVP group) and 6 patients who underwent mitral valve replacement and papillary muscle tugging approximation (MVR+PMTA group). The left ventricular ejection fraction was assessed by the modified Simpson method; the slope was assessed by the single-beat technique using transthoracic echocardiography. BNP levels were measured by chemiluminescent immunoassay.

Results: The left ventricular ejection fraction and slope did not significantly change from pre- to early post-surgery in the MVP+LVP group. Both the left ventricular ejection fraction and slope significantly increased 6 months after surgery in the MVR+PMTA group. Postoperative BNP level was low in the MVR+PMTA group. While the postoperative left ventricular ejection fraction did not correlate with BNP levels, the postoperative slope significantly correlated with BNP level after surgery in the MVP+LVP group and in the total functional mitral regurgitation group.

Conclusions: The change of slope was dependent on surgical procedures. In functional mitral regurgitation, the slope may be a more sensitive parameter in reflecting the left ventricular contractile function than the left ventricular ejection fraction. 


\section{Introduction}

It is difficult to precisely estimate the intrinsic left ventricular contractile function by left ventricular ejection fraction (LVEF) specially in patients with severely deteriorated heart. The LVEF is commonly used to reflect the cardiac systolic function because it can be measured easily and non-invasively using transthoracic echocardiography. However, the LVEF, as estimated by echocardiography, has some limitations. Firstly, the LVEF is strongly affected by afterload and the LVEF is under- and overestimated in high- (e.g. vasoconstriction) and low-afterload conditions (e.g. vasodilation or mitral regurgitation [MR]), respectively [1]. In functional MR (fMR) with low LVEF, the LVEF did not correlate with late outcomes after mitral valve repair, replacement, and catheter intervention using the MitraClip [2-4]. Furthermore, it is well known that patients who have normal LVEF can develop heart failure as a result of diastolic dysfunction (heart failure with preserved EF) [5].

The slope in the preload recruitable stroke work relationship (Mw) has been recognized as a load- and LV size-independent contractile functional parameter and an index of the overall ventricular function, reflecting not only systolic, but also diastolic properties. We have reported Mw, but not LVEF, can be a predictive ventricular functional parameter for survival after surgery on the dilated heart with MR [6, 7]. However, there have been no reports regarding the perioperative changes of $\mathrm{Mw}$, and it is currently unknown how the LV function recovers from surgical interventions for MR. Therefore, we investigated the change in cardiac contractile parameters (LVEF and Mw) before and after mitral surgery and their correlation with serum brain natriuretic peptide (BNP) levels, the most common biomarker for heart failure, in patients with fMR. 


\section{Methods}

\section{Study population}

This retrospective observational study was performed at a single center (Hokkaido University Hospital). The study included 16 patients who underwent mitral valve (MV) surgery for severe fMR with non-ischemic dilated myocardiopathy (NIDCM) excluding the effect of bypass surgery for ischemic cardiomyopathy. From 2006 to 2015, 27 patients with NIDCM underwent left ventriculoplasty (LVP) with MV annuloplasty, papillary muscle approximation (PMA), and suspension (PMS). Ten patients were included for analysis (MVP+LVP group) (Fig. 1). We changed our surgical strategy and developed a new method, called "papillary muscle tugging approximation (PMTA),” which is a MV replacement (MVR) combined with single PMA and PMS without LVP [8]. Six patients with NIDCM who underwent MVR+PMTA from 2015 to 2017 were included for analysis (MVR+PMTA group). All patients had refractory heart failure and were not considered to be suitable candidates for heart transplantation due to old age and/or personal refusal.

In order to compare perioperative changes in the LVEF and Mw, 10 patients with degenerative MR who underwent MVP and had adequate echocardiographic data to evaluate the Mw were included in this study as the degenerative group. The LVEF and Mw values in 10 patients (mean age, $60 \pm 6$ years; 5 men; LVEF, 62\% $\pm 3 \%$ ) without any echocardiographic abnormalities were also measured as a control. The University Ethics Committee approved the research protocol (No. 017-0433).

\section{Surgical procedures of MVP and LVP}

For the 8 patients with large LV diastolic dimension $(\geq 70 \mathrm{~mm})$ in the MVP+LVP group, 
an overlapping LVP without a patch was performed, as previously described [9]. A Batista-type operation was performed for the 2 patients with myocardial fibrosis only in the posterior part of the LV. The MVP was conducted as a part of our original mitral complex reconstruction for all 10 patients. Briefly, papillary muscles were approximated side-by-side from the basis to the heads using three pledgeted mattress sutures (complete PMA) [10]. A CV-3 expanded polytetrafluoroethylene (ePTFE) suture was placed between the site of the chordal attachment of the approximated papillary muscles and the middle of the anterior mitral annulus. This suture was then passed through the true-sized semi-rigid total annuloplasty ring (PMS) [11].

\section{Surgical procedures of MVR and PMTA}

The PMTA was a combination of MVR with chordal preservation, single PMA, and PMS, without LVP [8]. After left atriotomy, the anterior MV leaflet was divided into two parts at the middle which were then attached to both commissures by pledgeted 5-0 polypropylene sutures. The papillary muscles were then approximated side-by-side using one pledgeted 3-0 polypropylene mattress suture (single PMA). The PMS was performed in the same manner as for the LVP group. The MV was then replaced using a mechanical valve in the intra-annular position. We used a low-profile mechanical valve (Abbott Park, Illinois, USA).

\section{Assessment of cardiac function using echocardiography}

Commercially available ultrasonic systems including Vivid 7 or Vivid E9 (GE Healthcare, Milwaukee, WI, USA), Aplio XG or Aplio Artida (Toshiba Medical Systems, Tochigi, Japan), and Sonos 5500 or iE33 (Philips Ultrasound, Bothell WA, 
USA) were used for echocardiography. The examination was conducted by experienced examiners who were blinded to the study details. The following basic variables were measured from the parasternal long-axis view: LV end-diastolic dimension (LVDd) and end-systolic dimension (LVDs) (mm), interventricular septal thickness (IVST) (mm), and LV posterior wall thickness (LVPWT) (mm). On the apical long-axis image, the pulsed Doppler sample volume was located at the tip of MV leaflets in order to obtain the deceleration time (DcT) (ms) of the early transmitral flow. The LV end-diastolic volume (LVEDV), end-systolic volume (LVESV), and LVEF were measured using the modified Simpson method. The forward stroke and MR volumes were calculated by using pulsed wave Doppler at the LV outflow tract and MV annulus, respectively. Severe fMR was determined as follows: 1 ) an effective regurgitant orifice (ERO) $\geq 0.20$ $\mathrm{cm}^{2}$ and 2$)$ a regurgitant volume $\geq 30 \mathrm{~mL}$ (ERO $\geq 0.40 \mathrm{~cm}^{2}$, regurgitant volume $\geq 60 \mathrm{~mL}$ in degenerative MR) [12].

\section{Estimation of the slope in the preload recruitable stroke work relationship}

The Mw was estimated by using the formula described by Lee et al.: (total stroke work $) /[\mathrm{EDV}-\kappa \times \mathrm{EDV}+(1-\kappa) \times \mathrm{LV}$ wall $]\left(\mathrm{erg} \mathrm{cm}^{-3} \cdot 10^{3}\right)$ [13]. The EDV was derived from the total stroke volume divided by the LVEF. The LV wall was estimated by the echocardiography-derived LV mass. The constant, $\kappa$, was calculated as follows: $\kappa=0.004 \times \mathrm{LV}$ mass +0.6408 . We changed the method for calculating the stroke work from that we previously reported to calculate Mw both before and after surgery [6]. We calculated the total stroke work as follows: total stroke volumex mean blood pressure, which was previously calculated as follows: (total stroke volume $) \times($ SBP-LAP), because of the disadvantage in that we could not calculate Mw after MR disappeared. The 
previously reported cut-off value of 42 corresponds to about $35 \mathrm{erg} \mathrm{cm}^{-3} \cdot 10^{3}$ in the current study. The blood pressure was measured by a manometer. The total stroke volume was calculated as follows: forward stroke volume + MR volume $(\mathrm{mL})$.

\section{Measurement of serum brain natriuretic peptide}

Venous blood samples were collected in EDTA tubes after the patient had rested in the supine position. The BNP levels were measured immediately by using a chemiluminescent immunoassay method.

\section{Statistical analysis}

Echocardiography and BNP measurements were performed at 3 time points: baseline (before surgery), before discharge (early postoperative period), and 6 months after surgery (late postoperative period). Continuous variables are presented as means \pm standard deviation. For continuous variables, the unpaired t-test or Mann-Whitney’s U test were used to compare groups, as appropriate. One-way repeated measured analysis of variance (ANOVA) was used to compare pre- and post-operative and 6-month postoperative values. For categorical data, group differences were evaluated using the $\mathcal{\varkappa}^{2}$ or Fisher's exact test. A p-value $<0.05$ was considered statistically significant. Statistical analysis was performed with SPSS version 17.0 software (SPSS Inc., Chicago, IL, USA). 


\section{Results}

\section{Preoperative patient characteristics and echocardiographic parameters}

Table 1 shows the baseline clinical characteristics and echocardiographic data of each group. There were no significant differences between the fMR groups, except for male sex and the preoperative MR volume.

\section{Operative data}

Table 2 shows the operative data in each group. The operative, cardiopulmonary bypass, and aortic cross-clamp times were significantly shorter in the MVR+PMTA group than in the MVP+LVP group; this was presumably due to the absence of LVP in the MVR+PMTA group.

\section{Early outcomes}

The mean follow-up duration was $17 \pm 8$ months and $30 \pm 32$ months in the MVR+PMTA and MVP+LVP group, respectively. There were no patients who had more than mild residual MR in the MVP+LVP group in the latest follow-up. There was no mortality in the MVR+PMTA group, whereas there were 4 hospital deaths $(4,5,13$, and 14 months after surgery) in the MVP+LVP group (cardiac, 3; necrosis of the intestine, 1). The 6-month survival rates were $100 \%$ and $80 \%$ in the MVR+PMTA and MVP+LVP groups, respectively (log-rank $\mathrm{p}=0.07$ ).

\section{Perioperative changes of LVEF}

Fig. 2A shows the change in LVEF in each group. The perioperative LVEF did not significantly change in the MVP+LVP group. On the other hand, in the MVR+PMTA 
group, LVEF significantly increased at 6 months after surgery compared to baseline (p = 0.022 ) and early surgical period $(p=0.003)$. In the degenerative group, the LVEF was significantly decreased in the early surgical period compared to baseline $(\mathrm{p}=0.004)$ and was increased at 6 months after surgery compared to the early surgical period ( $\mathrm{p}<$ 0.001). Nonetheless, 6 months after surgery, the LVEF was comparable to that of the control group $(\mathrm{p}=0.41)$.

\section{Perioperative changes of $M w$}

Fig. 2B shows the change in the Mw in each group. The perioperative Mw did not significantly change in the MVP+LVP group. On the other hand, in the MVR+PMTA group, the Mw significantly decreased from baseline to the early surgical period ( $\mathrm{p}=$ 0.022) and increased from the early surgical period until 6 months after surgery $(\mathrm{p}=$ 0.038). In the degenerative group, the Mw also significantly decreased at the early surgical period $(p=0.020)$ and at 6 months after surgery $(p=0.017)$ from baseline. Nonetheless, the Mw 6 months after surgery was comparable to that of the control $\operatorname{group}(\mathrm{p}=0.12)$.

\section{Correlation between BNP and LV contractile functional parameters in fMR}

The BNP values were only applicable in the fMR groups. The BNP levels did not significantly change in the MVP+LVP group $(\mathrm{p}=0.20)$. On the other hand, in the MVR+PMTA group, the BNP levels tended to decrease consistently during the followup (522 \pm 310, $352 \pm 247$, and $166 \pm 147 \mathrm{pg} / \mathrm{mL}$ at baseline, early surgical period, and 6 months after surgery, respectively; $\mathrm{p}=0.08$ ). BNP level 6 months after operation was lower in the MVR+PMTA group than in the MVP+LVP group $(\mathrm{p}<0.01)$. 
Fig. 3A-C show correlation between the postoperative BNP levels and LVEF. The BNP levels did not correlate with the LVEF in any group. On the other hand, there was significant correlation between the postoperative BNP levels and the Mw in the MVP+LVP group (Fig. 3D $\left.\mathrm{R}^{2}=0.57, \mathrm{p}=0.005\right)$ and in the total fMR groups $($ Fig. 3F, $\left.\mathrm{R}^{2}=0.40, \mathrm{p}=0.001\right)$. The correlation between the Mw and the BNP levels in the MVR+PMTA group did not reach statistical significance (Fig. $3 \mathbf{E}$ ) possibly due to the small number.

\section{Discussion}

In the current study, we demonstrated that the Mw, but not the LVEF, correlated with serum BNP levels after mitral surgery in fMR. Thus, the Mw may be a more sensitive parameter, reflecting LV contractile function and heart failure, than the LVEF.

\section{Mw as a contractile functional parameter}

The concept of the preload recruitable stroke work (PRSW) relationship was first proposed by Glower et al. who used closed-chest dogs to demonstrate that the PRSW relationship was linear [14]. The Mw which was the slope of PRSW has been recognized as a load- and LV size-independent contractile functional parameter and an index of the overall ventricular function, reflecting not only systolic, but also diastolic properties. Although the PRSW was originally defined by invasive catheter examination, Lee et al. proposed a "single-beat technique” using transthoracic echocardiographic data and proved that the Mw could be estimated from a steady-state beat without alternation of the preload [13]. They further showed in subgroup analysis 
that the Mw derived from the single-beat technique closely matched the data gathered from the conventional catheter method in patients with different LV sizes, LV mass, and regional wall motion abnormalities.

\section{Correlation between BNP and Mw}

In the present study, there was significant correlation between BNP levels and Mw in the post-operative phase, although there was no correlation between BNP levels and LVEF. While the LVEF is a load-sensitive parameter reflecting only systolic function, Mw reflects both systolic and diastolic function; this is likely to explain why the BNP levels correlated only with the Mw. Thus, the Mw might be a more sensitive parameter to reflect heart failure than the LVEF. The preoperative BNP levels did not correlate with the preoperative Mw. We speculate that higher rates of inotrope use (MVP+LVP group, 50\%; MVR+PMTA group, 67\%) might have influenced the correlation before surgery in this cohort.

\section{Clinical implications of change in $M w$}

We previously reported that Mw, but not LVEF, predicted survival after MVP+LVP in patients with NIDCM [6]. However, we did not assess the Mw postoperatively routinely; thus, the benefit of surgical intervention was obscure. In the present study, the Mw significantly increased in the MVR+PMTA group, but not in the MVP+LVP group, 6 months after surgery. The LVP might have compromised LV function. Although longterm follow-up of more cases is required, the Mw may be used for the postoperative management of heart failure and the estimation of responders and non-responders for MVR+PMTA. 
The Mw also significantly decreased in the degenerative MR group in the early postoperative period. In a dog model of MR, when MR was induced at a constant preload, although forward stroke volume decreased, peak circumferential fibershortening velocity and peak contractile element velocity as barometers of LV contraction were significantly increased compared to the model without MR [14]. They argue that reduction of impedance to ejection allows the ventricle to empty further, which reduces ventricular wall tension and increases in the velocity of shortening. We also argue that patients with severe MR are in a hyper-contractile state before surgery with over-preload, and Mw decreases temporarily after surgery due to the elimination of MR which normalizes the pre- and afterloads.

\section{Changes of surgical strategy for NIDCM}

We changed surgical strategy from MVP+LVP to MVR+PMTA in 2015 because of unacceptable results of MVP+LVP as we previously reported [6]. There were two changes in the surgical approach for non-ischemic DCM with MR in 2015: 1) withdrawal from LVP, and 2) prevention of MR recurrence by the modified MVR. Warwick $\mathrm{R}$ et al. showed by finite element analysis that LVP (LV volume reduction) decreases forward stroke volume unless LVEF dramatically improves [16]. We can expect the increase in LVEF by LVP in ischemic DCM, but not in NIDCM. Furthermore, there has been little evidence showing that conventional chordal-sparing MVR improved symptoms and prolonged survival for a long time in NIDCM patients with severe MR. We expect additional effects of PM approximation and tugging to conventional chordal-sparing MVR, which needs to be validated in the future. 


\section{Limitations}

This study has several limitations. First, the number of subjects was small and the number of subjects who were not candidates for transplantation was quite limited; thus, the analysis of correlation in the MVR+PMTA group may have been underestimated. Second, a selection bias may exist in the MVP+LVP group as this was only comprised of patients with adequate echocardiographic data before and after surgery. However, there were no significant differences in the evaluated characteristics and survival rate between the included and excluded patients (data not shown). Third, the change of abnormal wall motion area after LVP might relate to the inaccuracy of the estimation of LV volumes and Mw which were derived from the biplane Simpson’s method. Forth, we selected mechanical valve in MVR to avoid interference with the subvalvular apparatus, to maximize the effective orifice area during exercise, and to obtain smaller transvalvular central leakage. However, mechanical valve has a risk of thrombus formation and we need careful follow-up.

\section{Conclusions}

The change of the Mw, which can be derived easily by the single-beat method, was dependent on the surgical procedures for fMR. The Mw, but not the LVEF, correlated with the serum BNP levels after surgery. Thus, in fMR, the Mw may be a more sensitive parameter reflecting LV contractile function than the LVEF. 


\section{Acknowledgements}

We would like to thank Editage for English language editing.

\section{Funding}

This research received no grant from any funding agency in the public, commercial, or not-for-profit sectors.

\section{Conflict of interest}

The authors declare that there is no conflict of interest. 


\section{References}

[1] Gaasch WH, John RM, Aurigemma GP. Managing Asymptomatic Patients With Chronic Mitral Regurgitation. Chest. 1995;108:842-7.

[2] Barth S, Hautmann MB, Kerber S, Gietzen F, Reents W, Zacher M, et al. Left ventricular ejection fraction of < 20\%: Too bad for MitraClip((c)) ? Cathete Cardiovasc Interv. 2017;90:1038-45.

[3] Braun J, van de Veire NR, Klautz RJ, Versteegh MI, Holman ER, Westenberg JJ, et al. Restrictive mitral annuloplasty cures ischemic mitral regurgitation and heart failure. Ann Thorac Surg. 2008;85:430-6.

[4] Maltais S, Schaff HV, Daly RC, Suri RM, Dearani JA, Sundt TM, 3rd, et al. Mitral regurgitation surgery in patients with ischemic cardiomyopathy and ischemic mitral regurgitation: factors that influence survival. J Thorac Cardiovasc Surg. 2011;142:9951001.

[5] Owan TE, Hodge DO, Herges RM, Jacobsen SJ, Roger VL, Redfield MM. Trends in Prevalence and Outcome of Heart Failure with Preserved Ejection Fraction. N Eng J Med. 2006; 355: 251-9.

[6] Shingu Y, Kubota S, Wakasa S, Ooka T, Kato H, Tachibana T, et al. Slope in preload recruitable stroke work relationship predicts survival after left ventriculoplasty and mitral repair in patients with idiopathic cardiomyopathy. J Cardiol. 2015;65:157-63. [7] Shingu Y, Ooka T, Katoh H, Tachibana T, Kubota S, Matsui Y. Feasibility and limitations of mitral valve repair, with or without left ventricular reconstruction in nonischemic dilated cardiomyopathy. J Cardiol. 2018;71:329-35.

[8] Matsui Y, Shingu Y, Wakasa S, Ooka T, Kubota S. Papillary Muscle Tugging Approximation for Functional Mitral Regurgitation. Ann Thorac Surg. 2019 in press. 
[9] Matsui Y, Fukada Y, Naito Y, Sasaki S, Yasuda K. A surgical approach to severe congestive heart failure--overlapping ventriculoplasty. J Card Surg. 2005;20:S29-34. [10] Wakasa S, Kubota S, Shingu Y, Ooka T, Tachibana T, Matsui Y. The extent of papillary muscle approximation affects mortality and durability of mitral valve repair for ischemic mitral regurgitation. J Cardiothorac Surg. 2014;9:98.

[11] Shingu Y, Yamada S, Ooka T, Tachibana T, Kubota S, Tsutsui H, et al. Papillary muscle suspension concomitant with approximation for functional mitral regurgitation. Circ J. 2009;73:2061-7.

[12] Baumgartner H, Falk V, Bax JJ, De Bonis M, Hamm C, Holm PJ, et al. 2017 ESC/EACTS Guidelines for the management of valvular heart disease. Eur Heart J. 2017;38:2739-91.

[13] Lee WS, Huang WP, Yu WC, Chiou KR, Ding PY, Chen CH. Estimation of preload recruitable stroke work relationship by a single-beat technique in humans. Am J Physiol Heart Circ Physiol. 2003;284:H744-50.

[14] Glower DD, Spratt JA, Snow ND, Kabas JS, Davis JW, Olsen CO, et al. Linearity of the Frank-Starling relationship in the intact heart: the concept of preload recruitable stroke work. Circulation. 1985;71:994-1009.

[15] Charles W. Urschel, James W. Covell, Edmund H. Sonnenblick, John Ross, Jr. Eugene Braunwald Myocardial mechanics in aortic and mitral valvular regurgitation: the concept of instantaneous impedance as a determinant of the performance of the intact heart. J Clini Invest. 1968;47:867-883.

[16] Warwick R, Pullan M, pullis M. Mathematical modelling to identify patients who should not undergo left ventricle remdeling surgery. Interact CardiovascThorac Surg. 2010;10:661-5. 


\section{Figure legends}

Figure 1. Flow chart for the inclusion process of the study. AVR, aortic valve replacement; LVP, left ventriculoplasty; MV, mitral valve; MVP, mitral valve plasty; MVR, mitral valve replacement; PMTA, papillary muscle tugging approximation.

Figure 2. Changes of the LVEF (A) and Mw (B) in the control, degenerative, MVP+LVP, and MVR+PMTA groups. 6Mo, 6 months after the operation; LVEF, left ventricular ejection fraction; LVP, Left ventriculoplasty; MVP, mitral valve plasty; MVR, mitral valve replacement; PMTA, papillary muscle tugging approximation; Pre-OP, before the operation; Post-OP, before discharge.

Figure 3. Correlations between postoperative BNP and LVEF (A-C), postoperative BNP and Mw (D-F) in MVP+LVP (A, D), MVR+PMTA (B, E), and total functional MR group (C, F). BNP, brain natriuretic peptide; fMR, functional mitral regurgitation; LVEF, left ventricular ejection fraction; LVP, Left ventriculoplasty; MR, mitral regurgitation; MVP, mitral valve plasty; MVR, mitral valve replacement; PMTA, papillary muscle tugging approximation. 
Table 1. Preoperative characteristics and echocardiographic data in each group

\begin{tabular}{|c|c|c|c|c|}
\hline \multirow[b]{2}{*}{ Variables } & \multirow{2}{*}{$\begin{array}{c}\text { Degenerative } \\
\text { MR } \\
(n=10)\end{array}$} & \multicolumn{3}{|c|}{ Functional MR } \\
\hline & & $\begin{array}{l}\text { MVP+LVP } \\
\quad(\mathrm{n}=10)\end{array}$ & $\begin{array}{c}\text { MVR+PMTA } \\
(n=6)\end{array}$ & $\begin{array}{c}\mathrm{p} \\
\text { value }\end{array}$ \\
\hline \multicolumn{5}{|l|}{ Demographic data } \\
\hline Age (y) & $59 \pm 15$ & $60 \pm 10$ & $60 \pm 12$ & 0.95 \\
\hline Male & $6(60 \%)$ & $9(90 \%)$ & $2(33 \%)$ & 0.036 \\
\hline Atrial fibrillation & $3(30 \%)$ & 7 (70\%) & $1(17 \%)$ & 0.12 \\
\hline Diabetes mellitus & $0(0 \%)$ & $4(40 \%)$ & $0(0 \%)$ & 0.23 \\
\hline Renal failure $(\mathrm{Cr}>2.0 \mathrm{mg} / \mathrm{dL})$ & $0(0 \%)$ & $1(10 \%)$ & $0(0 \%)$ & 1.00 \\
\hline Catecholamine dependent & $0(0 \%)$ & $5(50 \%)$ & $4(67 \%)$ & 0.63 \\
\hline NYHA class III/IV & $0 / 0$ & $8 / 2$ & $2 / 4$ & 0.12 \\
\hline $\mathrm{BNP}(\mathrm{pg} / \mathrm{mL})$ & $48 \pm 58$ & $1041 \pm 671$ & $522 \pm 310$ & 0.10 \\
\hline CRT device implantation & $0(0 \%)$ & $5(50 \%)$ & $1(17 \%)$ & 0.31 \\
\hline \multicolumn{5}{|l|}{ Echocardiographic data } \\
\hline LVDd (mm) & $56 \pm 5$ & $78 \pm 8$ & $71 \pm 11$ & 0.15 \\
\hline LVDs (mm) & $37 \pm 6$ & $69 \pm 9$ & $64 \pm 12$ & 0.30 \\
\hline LVEF (\%) & $63 \pm 12$ & $26 \pm 6$ & $23 \pm 8$ & 0.40 \\
\hline DcT (ms) & $186 \pm 35$ & $169 \pm 77$ & $166 \pm 19$ & 0.92 \\
\hline IVST (mm) & $9 \pm 2$ & $9.5 \pm 0.8$ & $7.8 \pm 2.3$ & 0.06 \\
\hline PWT (mm) & $8 \pm 2$ & $8.4 \pm 1.3$ & $7.7 \pm 1.6$ & 0.33 \\
\hline MR grade $(3 / 4)^{\dagger \dagger}$ & $3 / 7$ & $0 / 10$ & $2 / 4$ & 0.13 \\
\hline Forward stroke volume (mL) & $61.6 \pm 16.2$ & $46.3 \pm 13.6$ & $35.4 \pm 12.1$ & 0.12 \\
\hline MR volume (mL) & $58.7 \pm 20.8$ & $34.5 \pm 14.9$ & $59.9 \pm 23.1$ & 0.017 \\
\hline $\mathrm{Mw}\left(\mathrm{erg} \cdot \mathrm{cm}^{-3} \cdot 10^{3}\right)$ & $106 \pm 31$ & $35 \pm 7$ & $35 \pm 7$ & 0.98 \\
\hline Cardiac index $\left(\mathrm{L} / \mathrm{min} / \mathrm{m}^{2}\right)$ & $2.5 \pm 0.5$ & $1.9 \pm 0.5$ & $1.8 \pm 0.4$ & 0.68 \\
\hline LVEDV (ml) & $114 \pm 34$ & $283 \pm 97$ & $225 \pm 72$ & 0.23 \\
\hline LVEDVI $\left(\mathrm{ml} / \mathrm{m}^{2}\right)$ & $72 \pm 14$ & $169 \pm 53$ & $146 \pm 43$ & 0.40 \\
\hline LVESV (ml) & $47 \pm 25$ & $210 \pm 84$ & $176 \pm 67$ & 0.42 \\
\hline LVESVI $\left(\mathrm{ml} / \mathrm{m}^{2}\right)$ & $30 \pm 16$ & $125 \pm 14$ & $115 \pm 18$ & 0.67 \\
\hline
\end{tabular}

Values \pm standard deviation. ${ }^{\dagger}$ MVR+PMTA group vs MVP+LVP group. effective regurgitant orifice (ERO) $\geq 0.20 \mathrm{~cm}^{2}$, regurgitant volume $\geq 30 \mathrm{~mL}$ in functional MR; ERO $\geq$ $\underline{0.40 \mathrm{~cm}^{2}}$, regurgitant volume $\geq 60 \mathrm{~mL}$ in degenerative MR. ACE-I, angiotensin-converting enzyme inhibitors; ARB, angiotensin receptor blockers; BNP, brain natriuretic peptide; CRT, cardiac resynchronization therapy; DcT, deceleration time; IVST, interventricular septal thickness; LVDd, left ventricular end-diastolic dimension; LVDs, left ventricular end-systolic dimension; LVEF, left 
ventricular ejection fraction; LVEDV(I), left ventricular end diastolic volume (index); LVESV(I), left ventricular end-systolic volume (index); LVP, LV plasty; MR, mitral regurgitant; MVP, mitral valve plasty; MVR, mitral valve replacement; $\mathrm{Mw}$, the slope in the preload recruitable stroke work relationship; NYHA, New York Heart Association; PMTA; papillary muscle tugging approximation; PWT, posterior wall thickness. 
Table 2. Operative data

\begin{tabular}{lcccc}
\hline & Degenerative & \multicolumn{3}{c}{ Functional MR } \\
\cline { 3 - 5 } \multicolumn{1}{c}{ Variables } & MR & MVP+LVP & MVR+PMTA & p \\
& $(\mathrm{n}=10)$ & $(\mathrm{n}=10)$ & $(\mathrm{n}=6)$ & value $^{\dagger}$ \\
\hline Operation time (min) & $309 \pm 65$ & $440 \pm 22$ & $308 \pm 29$ & 0.003 \\
Cardiopulmonary bypass time (min) & $168 \pm 40$ & $251 \pm 67$ & $160 \pm 11$ & 0.006 \\
Aortic cross-clamp time (min) & $116 \pm 30$ & $129 \pm 21$ & $84 \pm 14$ & $<0.001$ \\
MAP ring size (mm) & $30 \pm 2.9$ & $29 \pm 2.3$ & - & - \\
MVR valve size (mm) & - & - & $28 \pm 1.6$ & - \\
Left ventriculoplasty (\%) & & & & - \\
Overlapping left ventriculoplasty & - & $8(80 \%)$ & - & - \\
Batista type & - & $2(20 \%)$ & & - \\
Total blood transfusion volume (mL) & $825 \pm 962$ & $2844 \pm 1005$ & $1852 \pm 1504$ & 0.13 \\
Concomitant procedures (\%) & & & & \\
Tricuspid annuloplasty & $1(10 \%)$ & $9(90 \%)$ & $6(100 \%)$ & 1.00 \\
Maze / Pulmonary vein isolation & $1(10 \%)$ & $7(70 \%)$ & $2(33 \%)$ & 0.30 \\
CRT device implantation & $0(0 \%)$ & $5(50 \%)$ & $1(17 \%)$ & 0.31 \\
Mechanical circulatory support (\%) & & & & - \\
IABP pre-OP & $0(0 \%)$ & $0(0 \%)$ & $0(0 \%)$ & - \\
IABP post-OP & $0(0 \%)$ & $3(30 \%)$ & $0(0 \%)$ & 0.16 \\
PCPS pre-OP & $0(0 \%)$ & $0(0 \%)$ & $0(0 \%)$ & - \\
PCPS post-OP & $0(0 \%)$ & $0(0 \%)$ & $0(0 \%)$ & - \\
\hline
\end{tabular}

Values \pm standard deviation. ${ }^{\dagger}$ MVR+PMTA group vs MVP+LVP group. CRT, cardiac resynchronization therapy; IABP, intra-aortic balloon pumping; LVP, LV plasty; MR, mitral regurgitation; MAP, mitral annuloplasty; MVR, mitral valve replacement; OP, operation; PCPS, percutaneous cardiopulmonary support; PMTA; papillary muscle tugging approximation 
Fig. 1

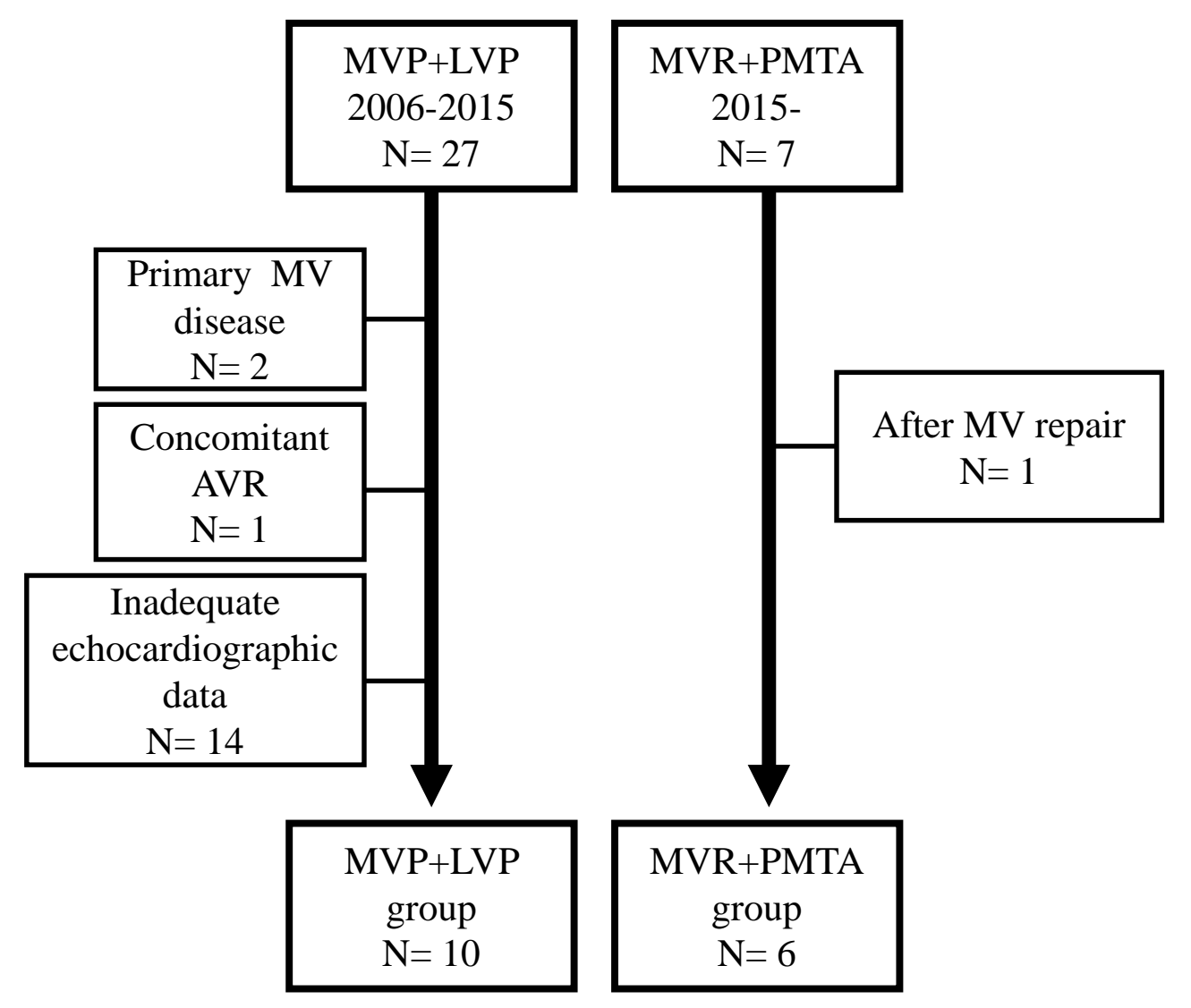




\section{Fig. 2}

\section{A}

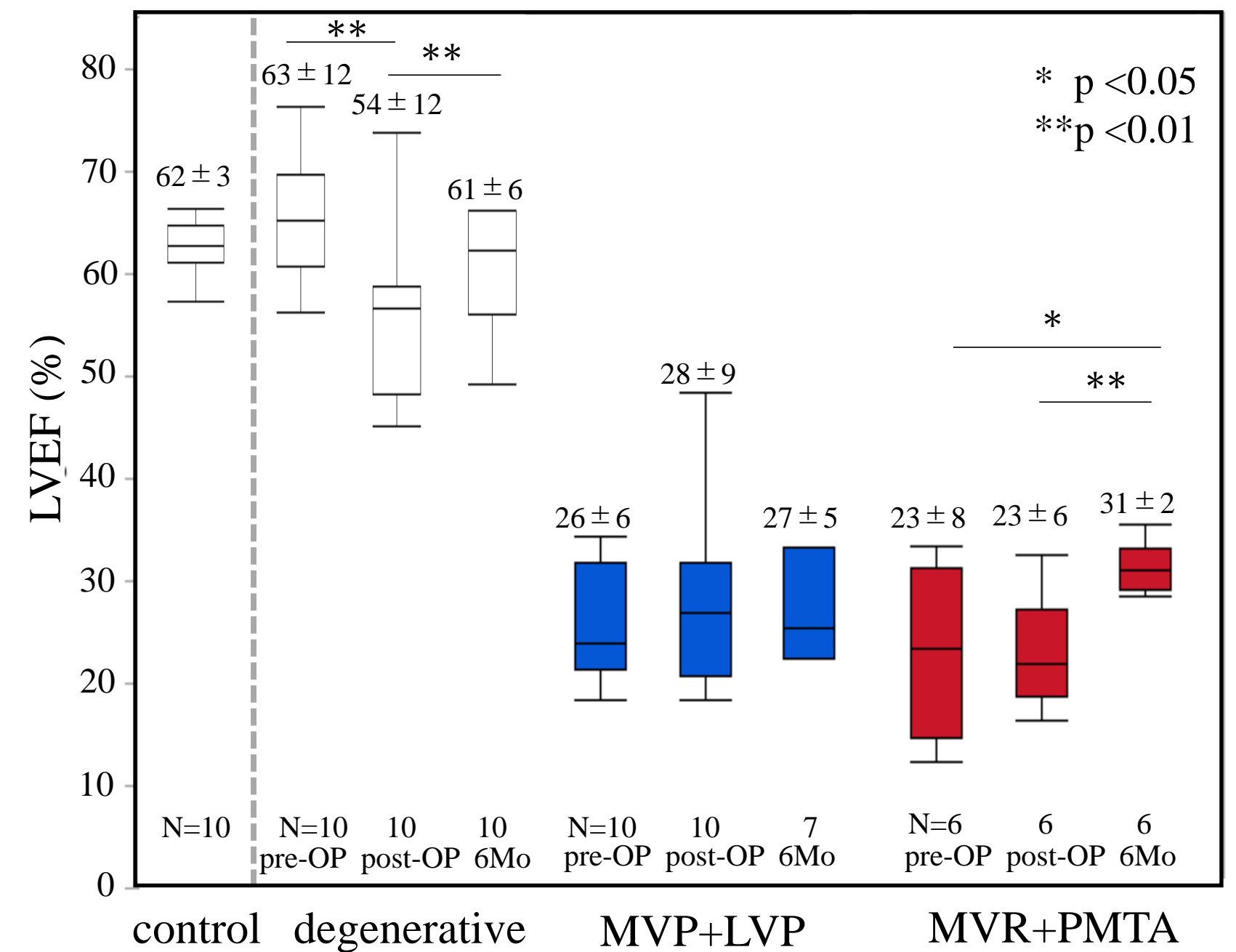

B

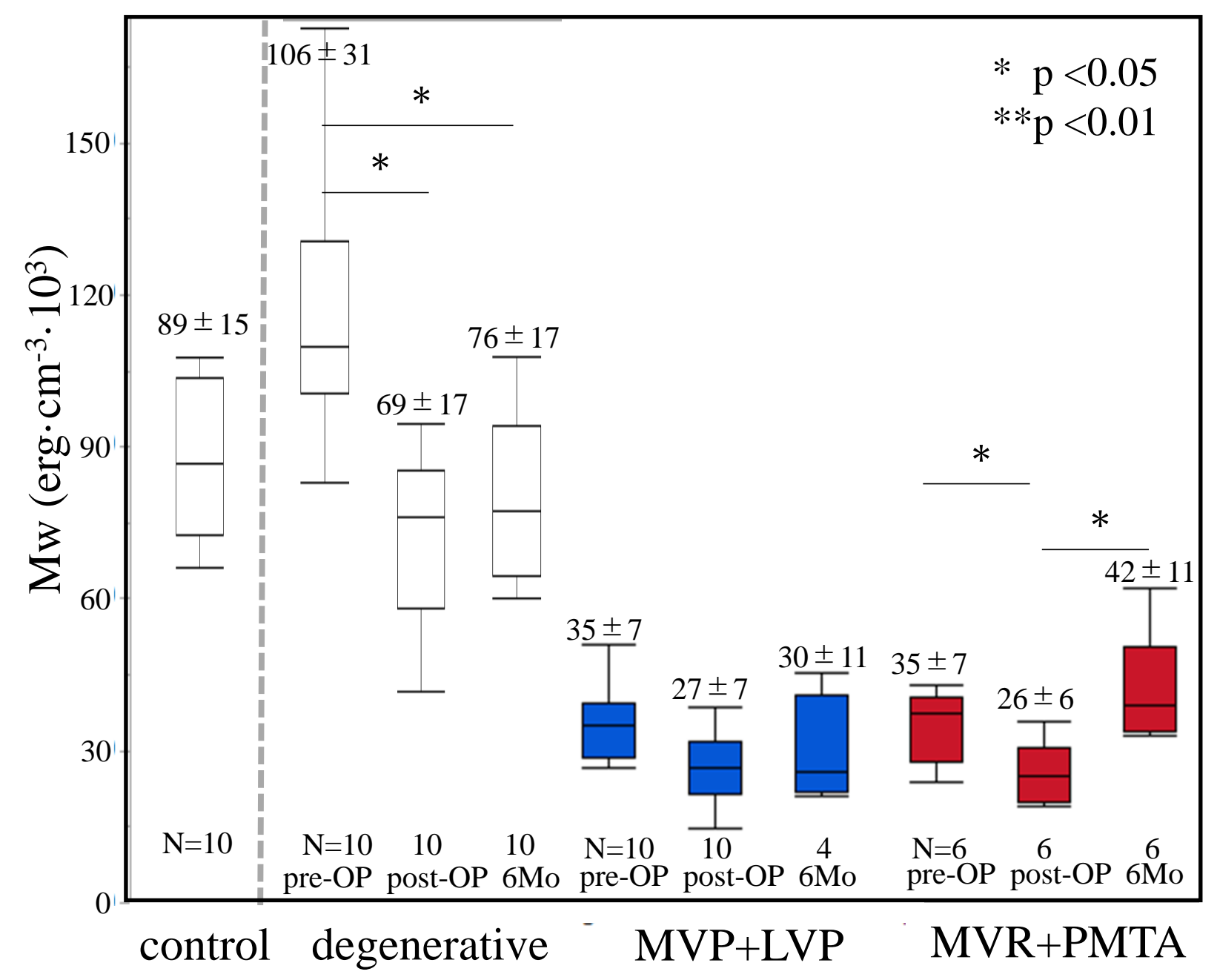




\section{Fig. 3}
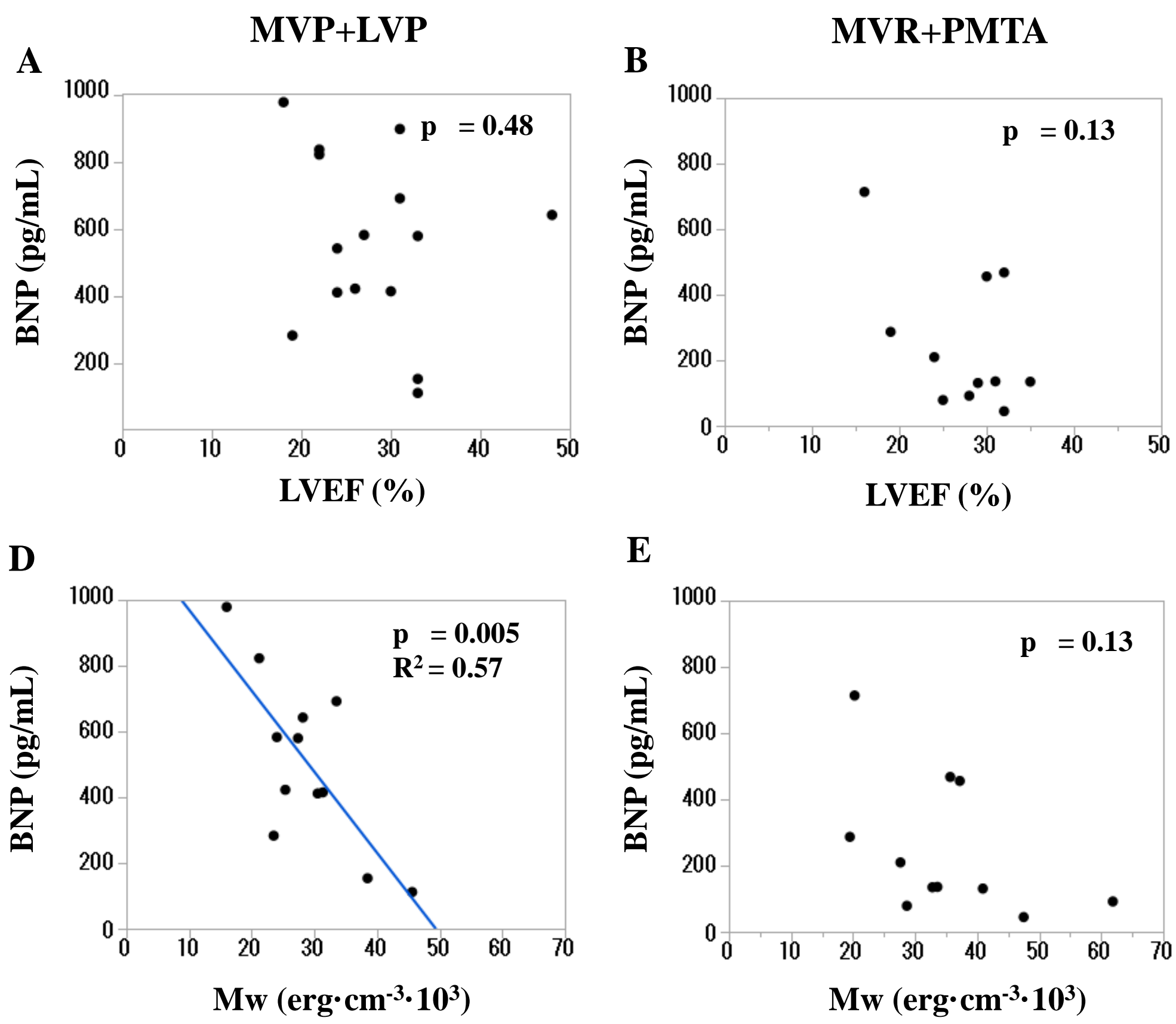

E
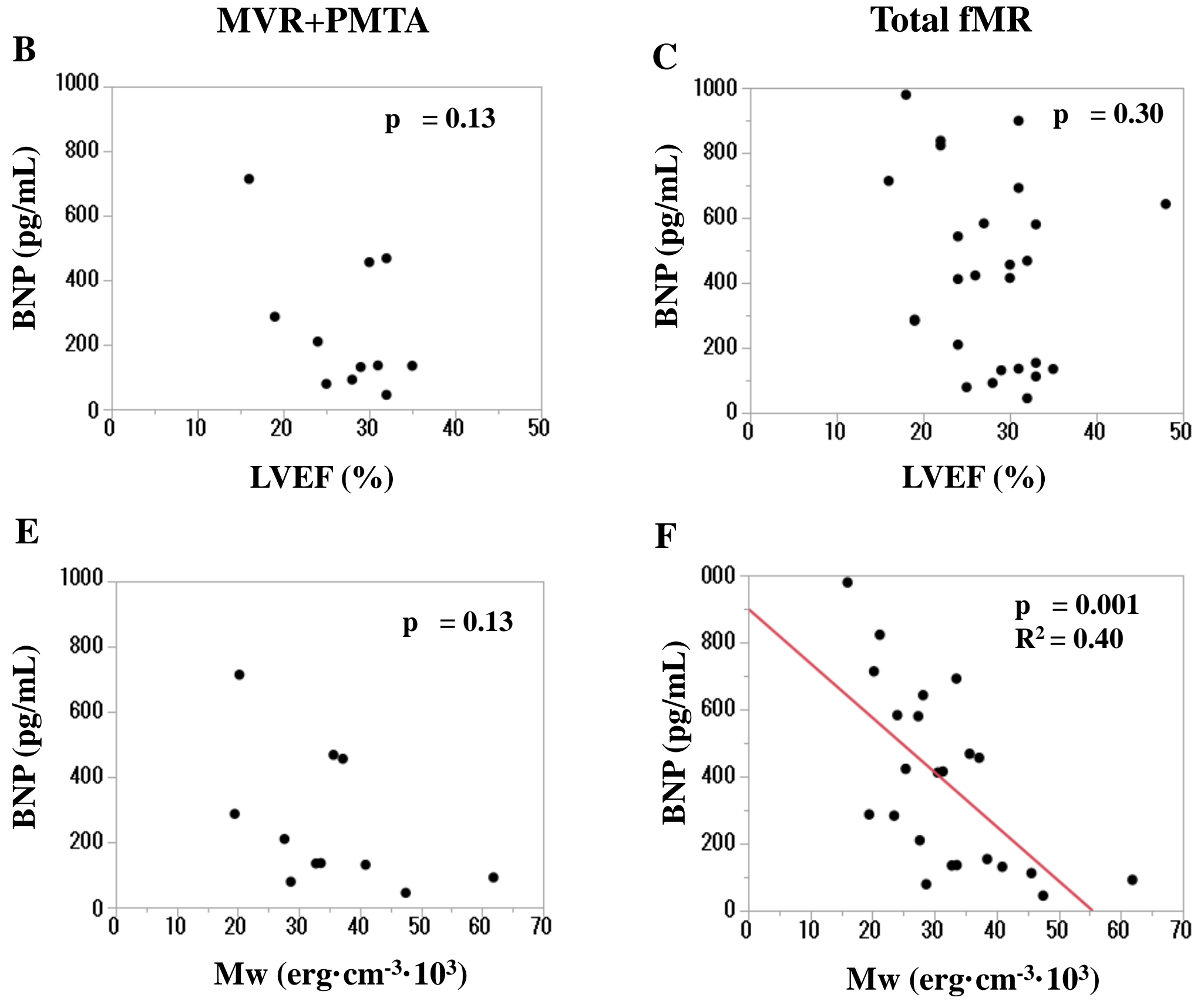